\title{
Genetic determinants of lipid profiles
}

Levels of lipoproteins and lipids in the blood are important risk factors for coronary artery disease (CAD) and metabolic syndrome. Three recent papers describe genome-wide association studies that identify a host of new and previously implicated variants that associate with lowdensity lipoprotein cholesterol (LDL-C), high-density lipoprotein cholesterol (HDL-C) and triglyceride levels, providing new potential targets for therapy.

High blood levels of LDL-C and of triglycerides are associated with an elevated risk of CAD; conversely, high levels of HDL-C are associated with a decreased risk. Moreover, high levels of triglycerides and low levels of HDL-C are seen in association with the metabolic syndrome.

Willer and Sanna et al. performed two genome-wide scans: one included 1,874 individuals from the FUSION study of type 2 diabetes; the other included 4,184 individuals from the SardiNIA study of ageing-associated variables. These data were combined with data on 2,758 individuals from the Diabetes Genetics Initiative (DGI). Together with analysis of $>10,000$ follow-up samples, their approach identified some 25 independent common variants that are associated with individual variation in lipid levels. Consistent with the low correlation between LDL-C, HDL-C and triglyceride levels, the authors found that distinct gene sets associate with each of these traits. In addition to confirming previously identified risk variants - such as those in the $A P O E-\underline{A P O C}$ cluster for LDL-C and CETP, LIPC and $L P L$ for HDL-C - novel risk alleles were identified at previously associated loci. The analysis also identified seven completely new loci. Although the functional connection between some of these variants and lipid metabolism remains unclear, some immediately fit the bill. For example, MLXIPL encodes a transcription factor that activates genes involved in triglyceride synthesis. As a final test, the authors confirmed that these variants associated with CAD itself by cross-referencing their results with data from the Welcome Trust Case Control Consortium.

Encouraged by their success, the authors set up a website that makes their data available to others (www. sph.umich.edu/csg/abecasis/public/ lipids). In fact, in the second paper, Kathiresan et al. combine their previously generated data from the DGI scan with the FUSION and SardiNIA studies to identify 18 independent loci that harbour variants (some of which are noncoding) that are associated with blood lipoprotein or lipid traits. Two new and five previously associated loci were identified for LDL-C, one new locus and six already known loci were found for HDL-C, whereas five new and four known

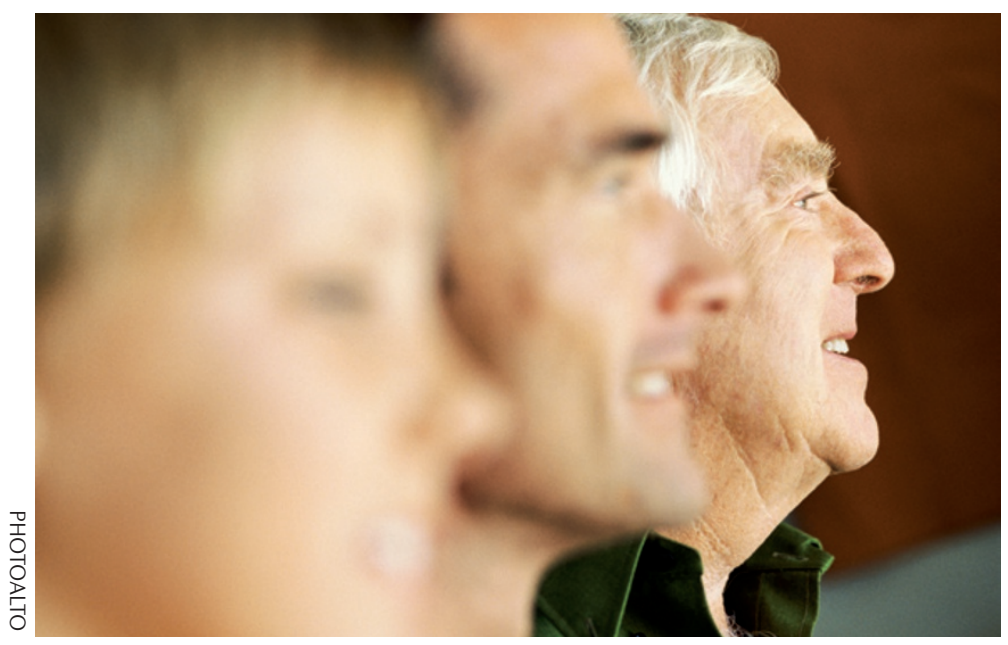

loci were found for triglycerides. The authors note an inverse relationship between the effect size of an allele and its frequency, and point out that, if deleterious, such alleles are not predicted to rise to a high frequency in a population.

In the third paper, Kooner and Chambers et al. report a three-stage association study in approximately 1,000 northern Europeans and 1,000 Indian Asians to identify variants that associate with lipid phenotypes in the metabolic syndrome. In agreement with the other two studies, they find that a polymorphism in $M L X I P L$ associates with HDL-C and triglyceride levels. Because of its function in lipogenesis and energy storage, the authors propose that MLXIPL might be a 'thrifty gene', which is advantageous when times of plenty are followed by a limited food supply.

Although variants that affect blood lipoprotein and lipid levels had been identified before, these studies have now made an impressive addition to this list, identifying several promising targets for therapy. Importantly, as discussed by Kathiresan et al., it is likely that only a small amount of the total variance in these traits is accounted for by SNPs that have been identified so far, highlighting the need for future studies to explain the remaining variance.

Magdalena Skipper

ORIGINAL RESEARCH PAPERS Willer, C. J. \& Sanna, S. et al. Newly identified loci that influence lipid concentrations and risk of coronary artery disease. Nature Genet. 13 January 2008 (doi:10.1038/ng.76)| Kathiresan, S. et al. Six new loci associated with blood low-density lipoprotein cholesterol, high-density lipoprotein cholesterol or triglycerides in humans Nature Genet. 13 January 2008 (doi:10.1038/ng.75)| Kooner, J. S. \& Chambers, J. C. et al. Genome-wide scan identifies variation in MLXIPL associated with plasma triglycerides. Nature Genet. 13 January 2008 (doi:10.1038/ng.2007.61) FURTHER READING Watkins, H. \& Farrall, M. Genetic susceptibility to coronary artery disease: from promise to progress. Nature Rev. Genet. 7 , 163-173 (2006)

WEB SITE

Genome Wide Associations Scans for HDL-C, LDL-C and triglycerides:www.sph.umich.edu/ csg/abecasis/public/lipids 